\title{
Etude biologique et écologique des Oligochètes aquatiques de la rivière Tambre et de ses milieux associés (Galice, Espagne)
}

\author{
E. Martinez-Ansemil ${ }^{1}$
}

Mots clés : Oligochètes, eaux courantes, répartition spatiale, populations, faunistique, Espagne.

Cette étude porte sur les communautés d'Oligochètes aquatiques vivant dans une petite rivière atlantique, le Tambre, et ses milieux associés (lagune, lac de barrage, ria). Elle concerne la répartition spatiale des différentes populations et l'analyse des principaux facteurs qui la déterminent.

Des données de l'évolution saisonnière des principales populations et des périodes de reproduction complètent l'inventaire faunistique des Oligochètes récoltés dans ces milieux.

Biological and ecological study of the aquatic oligochaetes of the River Tambre and its environment (Galicia, Spain).

Keywords : Oligochaetes, running waters, spatial distribution, population studies, faunistic, Spain.

This study describes communities of aquatic oligochaetes living in a small river flowing into the Atlantic, the River Tambre, and its environments (lagoon, reservoir, ria). It examines the spatial distributions of the different populations and analyses the chief factors responsible for these distributions.

Data on seasonal changes of the principal populations and their reproductive periods complete the faunistic study of oligochaetes found in these localities.

Parmi les différents aspects de l'étude des Oligochètes, ce sont la taxonomie, la faunistique, la reproduction et l'écologie dans les milieux pollués qui sont généralement abordés par les spécialistes. Les travaux sur leur biologie et leur écologie dans des milieux naturels non pollués sont assez rares.

Une étude physico-chimique des eaux du Tambre, rivière atlantique de basse altitude, a montré l'absence d'une véritable pollution (Lage-Yusty 1979). Ce cours d'eau peut être considèré comme oligotrophe comme en témoigne également la grande diversité de sa faune de Plécoptères (Membiela-Iglesia \& Martinez-Ansemil 1984). La présente étude sur les Oligochètes représente une contribution à la connaissance de ce groupe dans des milieux non encore trop altérés : la rivière Tambre et ses milieux associés (lagune, lac de barrage, ria).

1. Dpto. Bioloxia Animal, Univ. A Coruña, Campus da Zapateira $s / n ., 15071$ A Coruña, Espagne.

\section{Milieux et méthodes}

\subsection{Caractéristiques générales du milieu}

Le Tambre (fig. 1) coule en direction est-ouest, en Galice au nord-ouest de la Péninsule Ibérique. Il prend sa source à Sobrado dos Monxes (lat $\mathrm{N}=43^{\circ} 2^{\prime} 15^{\prime \prime}$; long $\mathrm{W}=$ $\left.4^{\circ} 19^{\prime} 30^{\prime \prime}\right)$ dans un petit lac artificiel situé à $510 \mathrm{~m}$ d'altitude et débouche dans la ria de Muros e Noia après un parcours d'environ $139 \mathrm{~km}$. Peu avant son embouchure le cours d'eau est barré par une retenue hydroélectrique (barrage de Barrié de la Maza).

Le bassin versant du Tambre, essentiellement granitique (fig. 1), a une superficie d'environ $1500 \mathrm{~km}^{2}$; les mouvements tectoniques (failles, plissements, fractures) ont contribué à la formation d'un vaste réseau hydrographique.

Les sols sont acides, riches en silice, en aluminium et en fer mais pauvres en calcium et en phosphore ( $c f$. Estudio Agrobiológico de la Provincia de La Coruña 1967).

Les diagrammes ombrothermiques de diverses localités du bassin versant sont présentés sur les figures 2 et 3 établies à partir du travail de Carballeira et al. (1983). 
Le climat est océanique avec des régimes thermique et pluviométrique réguliers.

Les débits sont maxima fin automne - début printemps et minima en été ou au début de l'automne. L'année 1979 fut particulièrement pluvieuse avec une importante sècheresse en été (fig. 4) : la différence entre le débit maximum (février) et le débit minimum (septembre) est très prononcée (fig. 4).

\subsection{Stations et méthodes d'étude}

\subsubsection{Choix et emplacement des stations}

13 stations d'échantillonnage, représentatives de la diversité des biotopes et des habitats, ont été choisies (fig. 1) :

St. 1 : lagune de Sobrado dos Monxes. Altitude = $510 \mathrm{~m}$. Trois points d'échantillonnage : 1 (A), 1 (B) et 1 (C) (fig. 6)

St. 2: Monte do Mazo, $3,9 \mathrm{~km}$ en aval de la st. 1 . Alt. $=480 \mathrm{~m}$.

St. 3 : Gosende, $16,3 \mathrm{~km}$ en aval de la st. 2. Alt. = $320 \mathrm{~m}$.
St. 4 : Ponte Carreira, $16,4 \mathrm{~km}$ en aval de la st. 3. Alt. $=280 \mathrm{~m}$.

St. 5: Sigüeiro, $28,4 \mathrm{~km}$ en aval de la st. 4 . Alt. $=$ $230 \mathrm{~m}$.

St. 6: Sigüeiro, $1,7 \mathrm{~km}$ en aval de la st. 5 . Alt. = $230 \mathrm{~m}$.

St. 7 : Sigüeiro, peu après la confluence avec le Lengüelle, $2 \mathrm{~km}$ en aval de la st. 6 . Alt. $=220 \mathrm{~m}$.

St. 8 : Ponte Albar, $17,2 \mathrm{~km}$ en aval de la st. 7. Alt. $=200 \mathrm{~m}$.

St. 9 : Ponte Maceira, $24 \mathrm{~km}$ en aval de la st. 8. Alt. $150 \mathrm{~m}$.

St. 10 : lac de barrage de Barrié de la Maza. Alt, = $100 \mathrm{~m}$. Deux points d'échantillonnage :

- 10 (A) :6,3 km en aval de la st. 9 ;

- 10 (B) : $5,4 \mathrm{~km}$ en aval du point 10 (A).

St. 11: Ria de Muros e Noia, $700 \mathrm{~m}$ en aval de la centrale hydroélectrique et à $11,5 \mathrm{~km}$ du point 10 (B). Alt. $=0 \mathrm{~m}$.

St. 12 : Ria de Muros e Noia, $1,1 \mathrm{~km}$ en aval de la st. 11. Alt. $=0 \mathrm{~m}$.

St. 13 : Ria de Muros e Noia, $1,2 \mathrm{~km}$ en aval de la st. 12. Alt. $=0 \mathrm{~m}$.

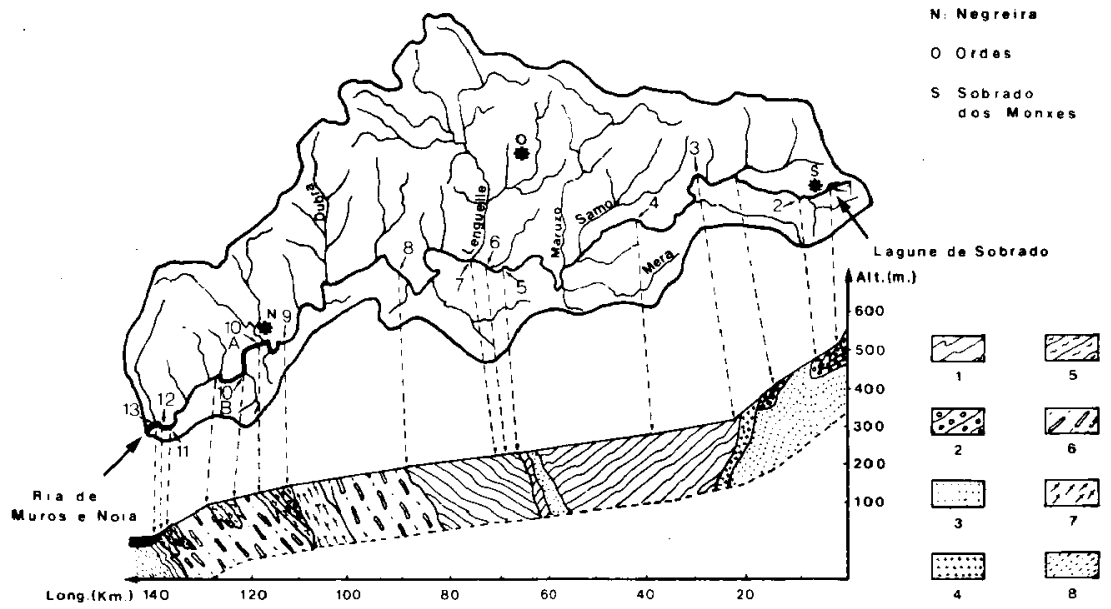

Fig. 1. Bassin versant du Tambre : nature géologique, réseau hydrographique, profil longitudinal et emplacement des stations. 1 : Roches sédimentaires (précambrien-cambrien) ; 2 : Gneiss oeillé ; 3 : Roches métabasiques (gabbros) ; $4:$ Granodiorite à biotite ; 5 : Injections magmatiques et migmatitiques ; $6:$ Granite porphyroïde à deux micas ; $7:$ Granite porphyroïde à biotite ; 8 : Gneiss granitique mylonitique. 


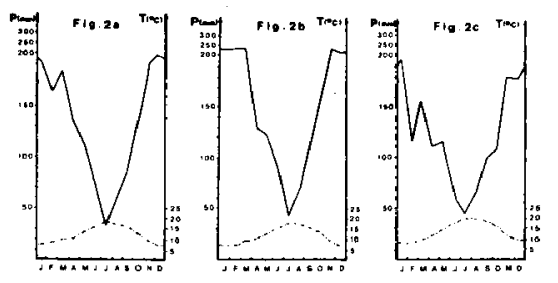

Fig. 2. Diagrammes ombrothermiques de Gaussen pour la période 1944-1974. 2a : à Présaras (cours supérieur); $2 \mathrm{~b}$ : à Labacolla (cours moyen) ; $2 c$ à Noia (cours inférieur).
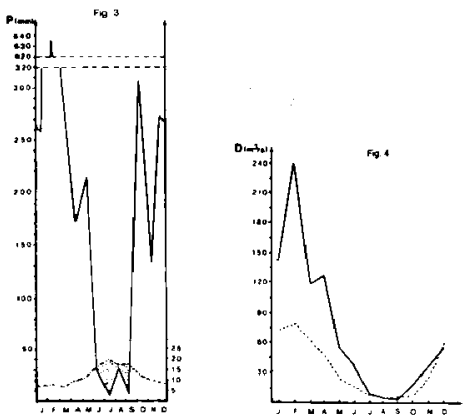

Fig. 3. Diagramme ombrothermique de Gatissen à Labacolla (cours moyen) pendant la période de récolte (année 1979).

Fig. 4. Variations du débit mensuel moyen du Tambre à proximité du lac de barrage. ( $\longrightarrow$; année $1979 ;(-.-)$ : période 1943-1960.

sécheresse dans le cours supérieur (décomposition des macrophytes) et en période de crues dans le cours inférieur (lavage de minéraux d'un chantier). Le pH est acide, l'amplitude thermique faible. Les eaux sont toujours saturées ou presque en $\mathrm{O}_{2}$.

- St. 7 : située juste en aval de la confluence avec le Lengüelle, c'est une zone profonde à courant faible dont le fond est recouvert d'argile et de vase riche en matière organique $(22,5 \%$ en décembre). L'exploitation de minéraux dans le bassin du Lengüelle provoque une forte turbidité et une augmentation de la conductivité en période de crue.

- Lagune (st. 1) : plus de $75 \%$ de la superficie sont recouverts par la végétation (fig. 6) ; le fond est formé d'une épaisse couche de vase riche en matière organique ( $29 \%$ dans le secteur C) qui dégage une forte odeur d' $_{2} \mathrm{~S}$ en été au niveau du secteur $\mathrm{C}$. En période estivale il existe une stratification thermique plus marquee dans le secteur $\mathrm{C}$ à végétation dense (plus de $8^{\circ} \mathrm{C}$ de différence entre la surface et le fond en aoùt). La teneur en $\mathrm{O}_{2}$ des eaux du fond varie peu en été : environ $2,5 \mathrm{mg} / \mathrm{l}$ ( $25 \%$ de saturation). La conductivité et surtout le $\mathrm{pH}$ fluctuent de façon importante ( $\mathrm{pH}=3,8$ à 9,4 en surface dans le secteur B). La transparence au disque de Secchi varie entre $1,4 \mathrm{~m}$ en été et $4,6 \mathrm{~m}$ en hiver (profondeur maxima de la lagune).

\section{- St. 2, 3, 4, 5, 6, 8, 9 : ce sont des milieux lotiques avec, surtout dans les trois premières stations, une grande diver sité de biotopes. Les eaux sont peu minéralisées : la conductivité ne dépasse $75 \mu \mathrm{S} / \mathrm{cm}$ qu'en période de mètres chimiques ont été mesurés à $10 \mathrm{~cm}$ sous la surface ; effectuées près du fond. Les principaux résultats, pés dans le tableau I, permettent de distinguer 5 grands types de milieu :}

Au cours de l'année 1979, nous avons déterminé cha-
que mois les principaux paramètres morphométriques, physiques et chimiques de chaque station :

- largeur et profondeur du lit ;

- végétation et granulométrie du substrat ; chi dans les milieux lénitiques, oxygène dissous (par la methode Winkler), pH et conductivité ;

- de façon plus ponctuelle, la teneur de l'eau en $\mathrm{Ca}$, sorption atomique ; la teneur en matière organique des 

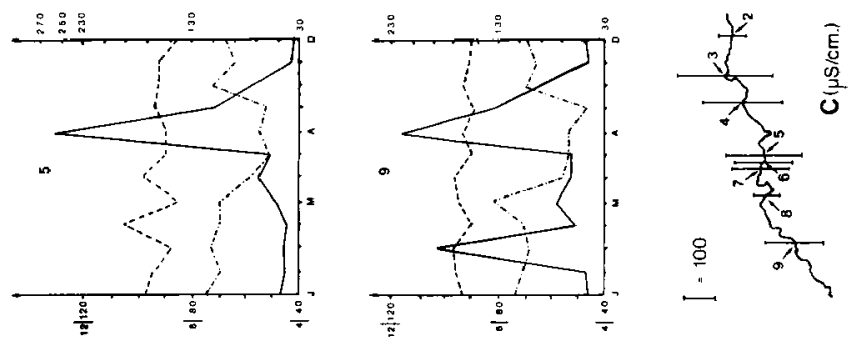

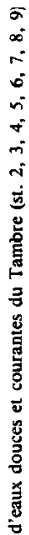
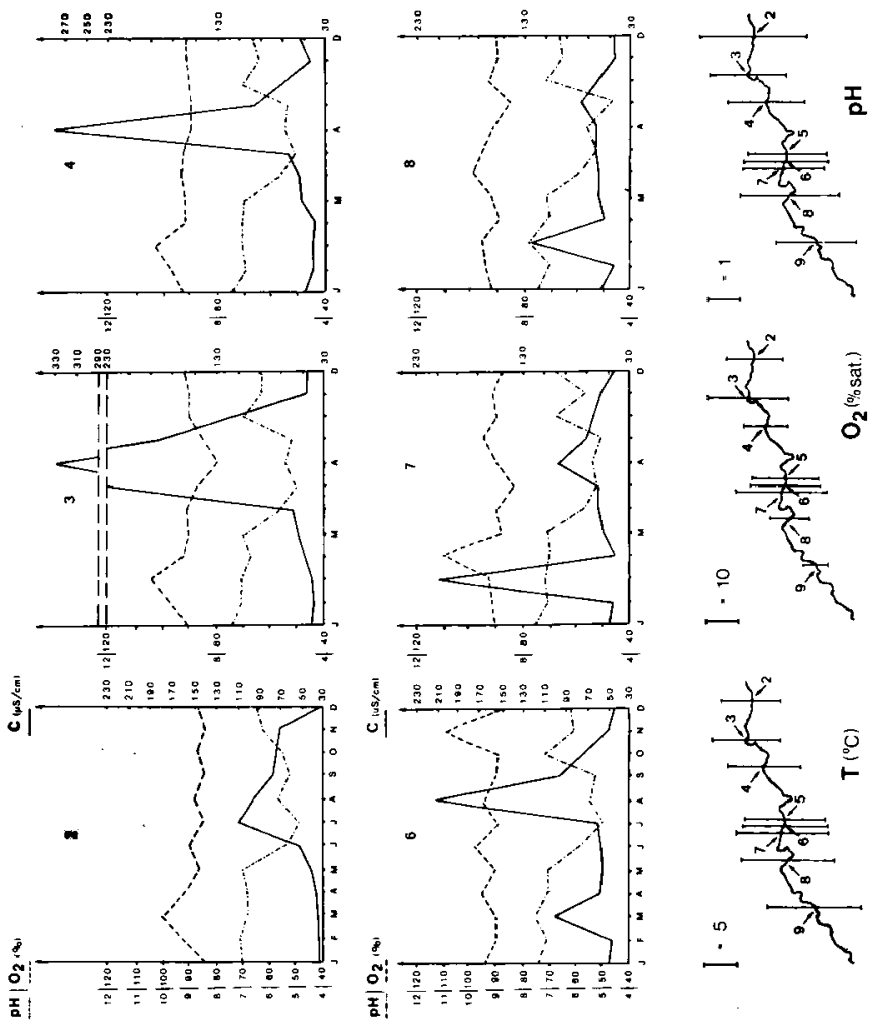

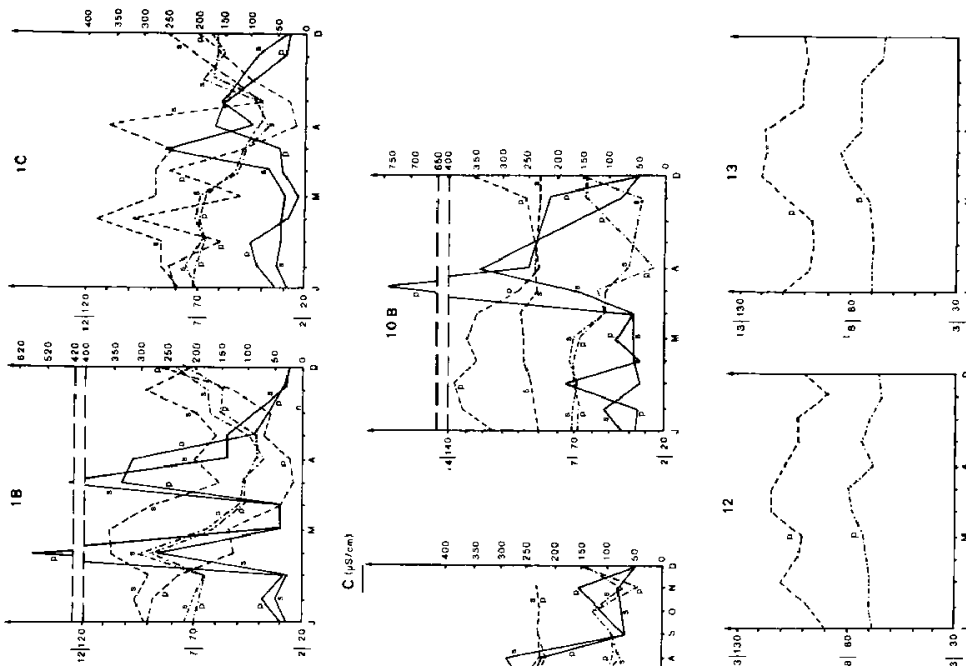

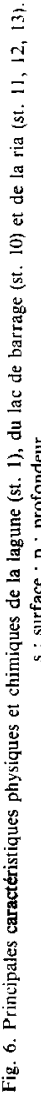
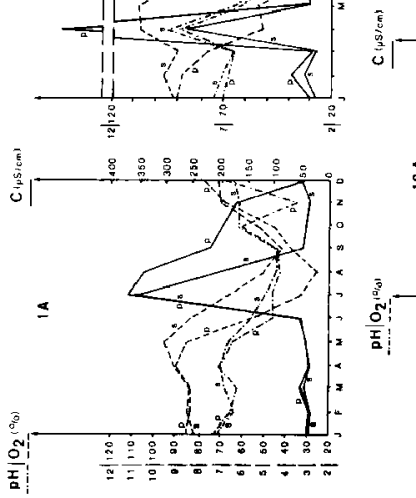

总品兽品
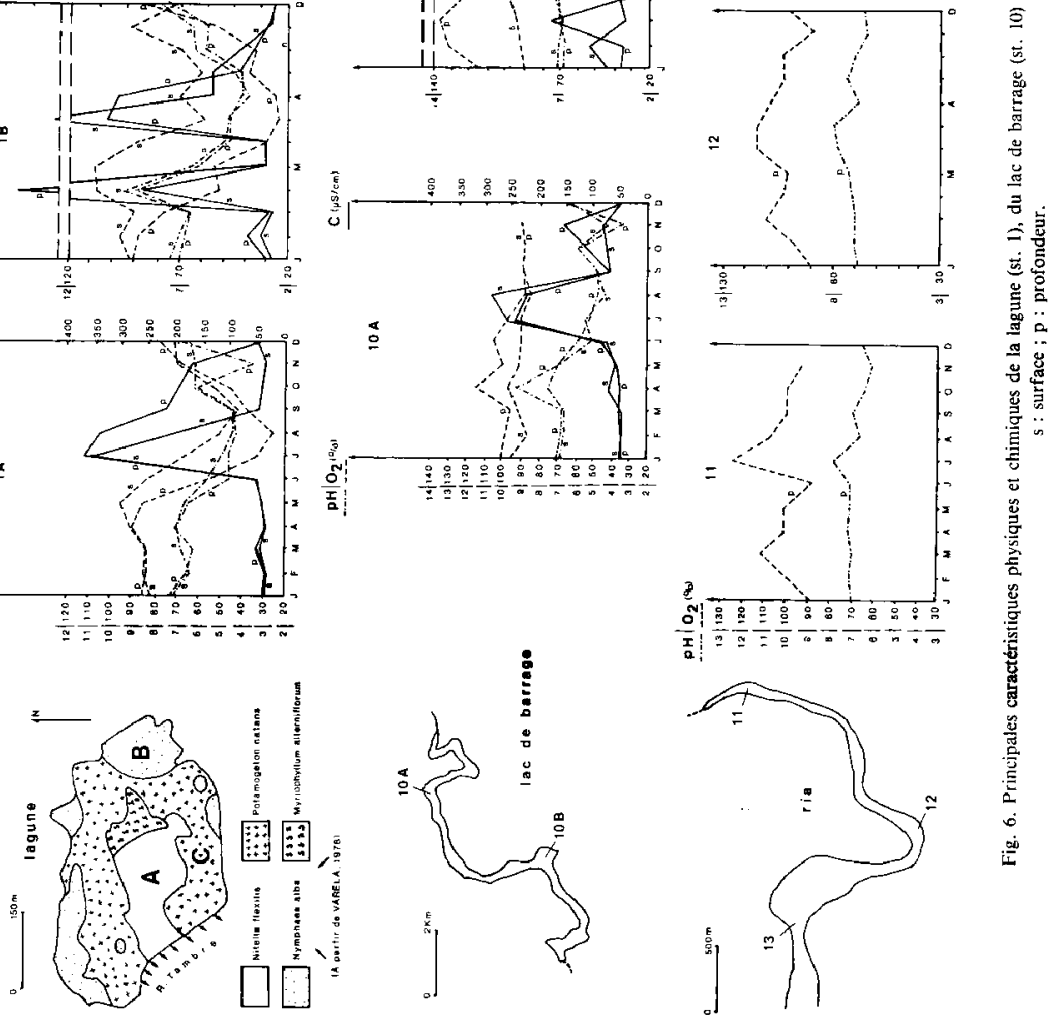
Tableau I. Principales caractéristiques morphométriques, physiques et chimiques des stations d'échantillonnage. $s$ : surface ; $f:$ fond $;{ }^{*},{ }^{* *},{ }^{* *}:$ abondance par ordre croissant.

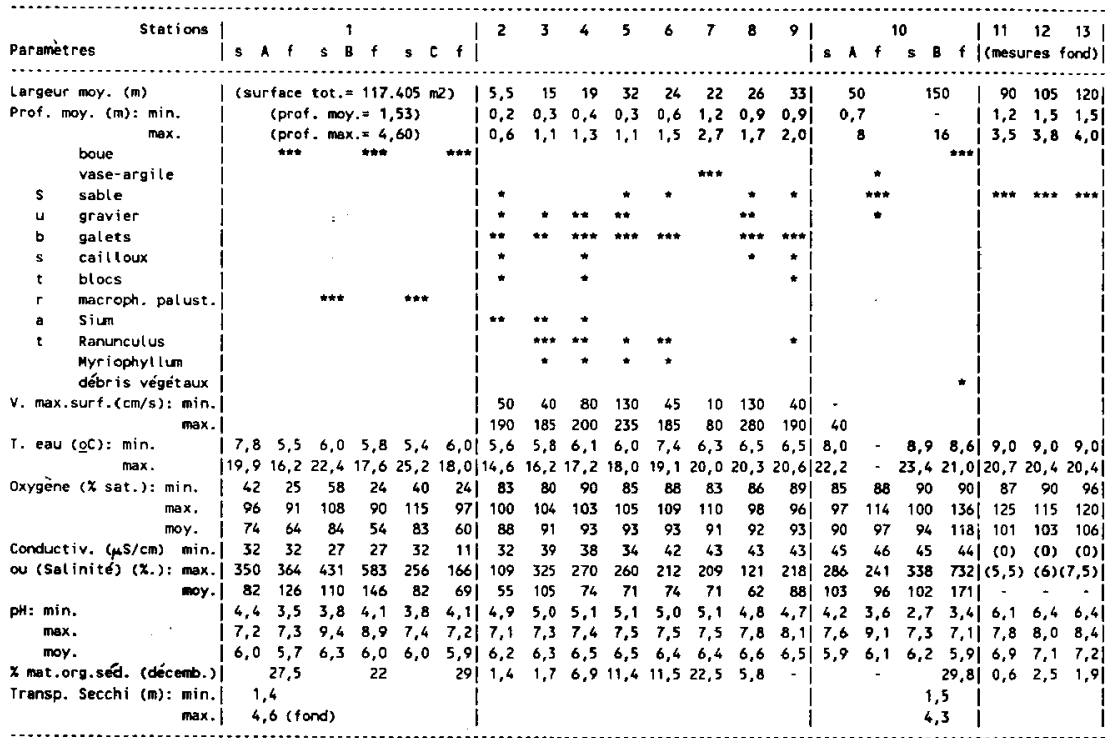

- Lac de barrage (st. 10). Il n'y a pas de véritable stratification thermique. Le $\mathrm{pH}$ est fortement acide en été : jusqu’à 2,7 en surface dans la zone B. La conductivité, faible en hiver $(50 \mu \mathrm{S} / \mathrm{cm})$, augmente de façon importante en été (jusqu'à $732 \mu \mathrm{S} / \mathrm{cm}$ ). Le sédiment est riche en matière organique $(29,8 \%)$ mais le brassage de la colonne d'eau et le fonctionnement du barrage (prise d'eau en profondeur) assurent une importante oxygénation des eaux du fond. La transparence varie entre $4,3 \mathrm{~m}$ en hiver et $1,5 \mathrm{~m}$ en été. Le marnage important empêche l'installation de macrophytes sur les bords.

- Ria (st. 11, 12, 13) : en période de crue ou à marée basse les eaux de la station 13 sont douces (jusqu'à $44 \mu \mathrm{S} / \mathrm{cm}$ de conductivité) alors qu'en été ou à marée haute elles atteignent 7,5\% de salinité ; les éclusées du barrage accentuent ces fluctuations et contribuent à l'instabilite du fond. Les eaux sont toujours saturées ou presque en $\mathrm{O}_{2}$. Le pH est proche de la neutralité et les variations les plus importantes ont été notées à la station $13(\mathrm{pH}=5,7$ à 8.4$)$. L'amplitude thermique ne dépasse guère $12^{\circ} \mathrm{C}$. La teneur en matière organique du sédiment est faible, notamment à la station 11 qui est soumise à l'extraction fréquente de sable.

\subsubsection{Prélèvements de faune.}

Chaque 2 mois, 2 à 6 prélèvements de faune selon la diversité des biotopes, ont été effectués à chaque station durant l'année 1979. En fonction de la vitesse du courant et de la profondeur nous avons utilisé soit un filet Surber $\left(500 \mathrm{~cm}^{2}\right)$, soit une benne Ekman, soit des carottiers en altuglass de 4 ou $8 \mathrm{~cm}$ de diamètre ; les Oligochètes vivant sur la végétation de la lagune ont été récoltés à l'aide d'un filet troubleau. Le vide de maille des différents filets utilisés était de $250 \mu \mathrm{m}$. 


\section{Inventaire faunistique et évolution sai- sonnière des populations}

Il existe une grande diversité spécifique (Tableau II), liée à l'hétérogénéité des milieux prospectés ; la majorité des 68 espèces recensées sont des formes cosmopolites ou à large répartition géographique. La richesse spécifique est élevée dans les stations d'eau courante (4l espèces à la st. 4) et, à un degré moindre ( $26 \mathrm{sp}$.), dans le barrage alors que la lagune (14 sp.) et la ria (11 sp.) hébergent un peuplement assez pauvre.

\section{Lumbriculidae}

La plupart des 16 spécimens matures de $S$. brachystylus ont été collectés en automne et en hiver ( 4 le $28 / 01,2$ le $31 / 07,1$ le $30 / 09$ et 9 le $28 / 11$ ) ; dans le Centre et l'Est de l'Europe des individus matures ont été souvent signalés au printemps ou en été (Kasprzak 1973, Timm 1979). Giani (comm. or.) a observé des individus matures tout au long de l'année dans le Laudot (S-O Massif Central, France).

Des $S$. heringianus matures ont été récoltés lors de chaque prélèvement mais presque la moitié des 432 spécimens ont été capturés en octobre et novembre. Une période de maturité étalée sur toute l'année, avec des maximums d'abondance à différentes périodes selon les conditions du milieu, a été souvent signalée pour cette espèce (Juget 1967, Timm 1967, 1979, Thomas et al. 1979).

Nous avons récolté des $S$. parvus matures tout le long de l'année excepté en août ; plus de $70 \%$ des 132 individus ont été collectés en novembre et février. Les données sur la reproduction de cette espèce sont rares : Cernosvitov (1928) et Giani \& Martinez-Ansemil (1981) trouvent des individus sexuellement développés en plein été.

\section{Naididae}

1 seul individu mature de $C$. crystallinus a été capturé à la station 12 le 10/06. Plus de $80 \%$ des $C$. diastrophus ont été récoltés en octobre et l'abondance de cette espèce était minimale en été. Près de $70 \%$ des C. langi ont été récoltés en avril. La plupart des $C$. langi ont été collectés dans le lac de barrage et les $C$. diastrophus uniquement en eau courante ;
A. leidigii est uniquement présent dans le lac de barrage. Sur 170 individus, 163 ont été récoltés au mois d'avril, seulement 3 en octobre et 4 en février. Ces résultats sont en accord avec les observations de Kasprzak \& Szczesny (1976), Frenzel (1981) et Loden (1981) alors que Pfannkuche (1981) a également observé un maximun d'abondance au début de l'automne. Près de $40 \%$ des individus récoltés étaient matures ( 3 en février et 63 en avril). Loden (op. cit.) constate également l'apparition d'individus matures lors du pic d'abondance précédant l'arrêt de la reproduction asexuée.

A. sannio n'a été récoltée qu'à l'automne (34 individus en octobre et 1 en novembre). $S$. josinae a été collectée tout le long de l'année mais elle est plus abondante au printemps. 1 seul individu mature de $U$. uncinata a été observé le $06 / 10$; cette espèce est souvent mature en été (Piguet \& Bretscher 1913, Timm 1970).

N. alpina représente près du tiers du total des Oligochètes récoltés ; elle est surtout abondante en automne : $60 \%$ des individus ont été capturés en octobre aux stations $4,5,6,8$ et 9 . Les 35 spécimens matures prélevés début juin ne représentent que $4 \%$ des effectifs récoltés à cette période quì prècède le minimum d'abondance (été). Dans la rivière Cynon (Angleterre), la période de maturité (été) coïncide avec le pic d'abondance maximale (Learner et al. 1978) alors qu'elle lui fait suite dans des ruisseaux des Carpathes (Dumnicka 1982).

$N$. communis est abondante tout le long de l'année ; cependant, ses effectifs augmentent légèrement en hiver et diminuent fortement en été. Cette espèce cosmopolite et ubiquiste a une grande capacité d'adaptation de son cycle de développement aux conditions du milieu : sa période d'abondance maximale est variable (Wachs 1967, Learner et al. 1978, Loden 1981, Smith 1985a).

$N$. elinguis est abondante au printemps et faiblement représentée le reste de l'année dans les trois stations de la ria (fig. 7). Nous avons récolté 6 individus matures ( 1 à la st. 11 et 5 à la st. 13) le 10/06 lors du pic d'abondance mais ils ne représentaient qu'un faible pourcentage de l'effectif total. A la station 13 où les fluctuations de salinité sont importantes la population disparaît après le pic d'abondance. Une dynamique de population tout à fait comparable, bien que décalée dans le temps (pic d'abondance en mars) a été observée dans une rivière 
méditerranéenne (l'Argens, France) par Giani \& Martinez-Ansemil (1981). Les périodes de recrutement de $N$. elinguis sont généralement bien définies mais varient selon les milieux (Eyres et al, 1978).

$N$, pardalis semble être surtout abondante à la fin de l'hiver ; sur 176 individus aucun n'a été récolté en juin et août. Wachs (1967), Learner et al. (1978, 1982) signalent des maximums d'abondance pendant la période chaude et Loden (1981) à l'automne.

Les populations de $N$. variabilis ne varient pas numériquement au cours de l'année ce qui est en accord avec les observations de Piguet (1906). Cependant, des variations annuelles avec des maximums d'abondance ont été observés (Loden 1981, Pfannkuche 1981).

Plus de $50 \%$ des 244 S. appendiculata ont été récoltés fin novembre et une forte diminution des effectifs semble avoir lieu en été où le nombre de spécimens recensés représente moins de $10 \% \mathrm{du}$ total.

En eau courante, $V$. comata présente un maximum d'abondance a l'automne alors que la population de la lagune (st. 1) est stable tout le long de l'année.

L'eutrophisation de la lagune pourrait être à l'origine de la brusque diminution des effectifs de $S$. lacustris pendant la période chaude : seulement $0,5 \%$ des 2065 individus ont été récoltés à la fin du printemps et en été contrairement aux observations de Berg (1938), Timm (1967) et Smith (1986).

Plus de $80 \%$ des $245 P$, osborni ont été capturés fin novembre ; aucun spécimen n'était présent dans les prélèvements du mois d'août.

\section{Remarques sur P. osborni :}

Loden \& Harman (1980) suggèrent une éventuelle synonymie entre $P$. rosea et $P$. sima alors que Smith (1985b) pense plutôt à une synonymie entre cette dernière espèce et $P$. osborni. Les soies aciculaires des $P$. osborni et des $P$. sima que nous avons récoltées diffèrent fortement (cf Martinez-Ansemil \& Giani 1982, figs 6 et 7) ; les dents intermédiaires des soies aciculaires de $P$. sima sont bien visibles au microscope optique aussi bien pour les spécimens d'eau douce (st. 4) que pour celui d'eau saumâtre (st. 12). Aucun individu de $P$. rosea n'a été identifié.

\section{Tubificidae}

Parmi les $732 T$. ignotus récoltés, 60 étaient à l'état mature ( 8 en juin, 50 en août et 2 en novembre). Un maximum d'abondance a eu lieu en été lors de la période de maturité. Des individus matures de cette espèce ont été observés en été (Berg 1948, Laakso 1969a, Brinkhurst 1964). Giani (1984) trouve des individus sexuellement développés en juin et décembre lors des maximums d'abondance; Ladle (1971) constate des pics de maturité et d'abondance en hiver.

Seulement 30 individus matures de $T$. tubifex ont été récoltés ; la reproduction de cette espèce s'étale sur toute l'année avec une baisse d'intensité pendant la période chaude (aucun individu en juin). Une période de maturité étalée sur toute l'année a déjà èté signalée pour cette espèce (Brinkhurst \& Kennedy 1965, Timm 1967, Pfannkuche 1981, Giani 1984) ainsi qu'une reproduction moins intense en été (Ladle 1971, Young 1974).

La plupart des 200 individus matures de $L$. hoffmeisteri ont été récoltés dans la zone profonde du lac de barrage (st. 10), tout au long de l'année avec un pic en été (fig. 7). Le cycle de vie de $L$. hoffmeisteri est très variable (Kennedy 1966, Poddubnaya 1980, McMurray et al. 1982), des individus matures sont généralement trouvés tout le long de l'année avec des pics d'abondance plus ou moins marquées à différentes périodes.

Nous avons récolté $\mathrm{i}$ seul spécimen mature de $L$. udekemianus (le 10/10), de P. albicola (st. I le $28 / 01$ ), de $B$. vejdovskyanum (st. 10 le $05 / 04$ ), de $R$. subterraneus (st. 6 le $05 / 06$ ) et de $R$. coccineus (st. 2 le 27/01).

La période d'abondance maximale de $S$. velutinus s'étale de la fin du printemps à la fin de l'automne ; parmi les 707 individus récoltés 13 étaient matures (4 en février, 1 en août, 1 en octobre et 7 en décembre).

$32 R$. falciformis matures ont été récoltés (15 le $27 / 01,15$ le $31 / 03$ et 2 le $03 / 06$ ). $65 P$. tourenqui matures ont été observés tout le long de l'année : 5 en février, 19 en juin, 13 en août, 10 en octobre et 18 en décembre.

A. monospermathecus n'est bien représentée qu'à la station 12 ou elle est abondante de la fin du printemps au début de l'automne lorsque la température et la salinité sont élevées; elle disparaît 

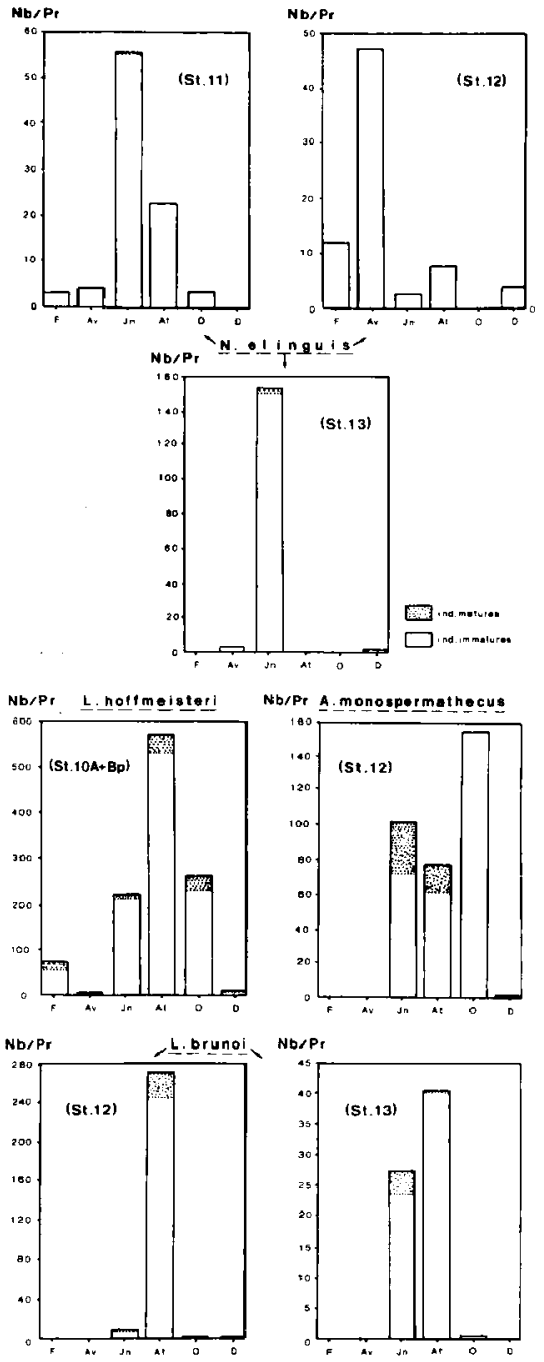

Fig. 7. Evolution numérique des populations de plusieurs espéces à différentes stations pendant la période de recolte. $\mathrm{Nb} / \mathrm{Pr}$ : nombre d'individus par prélèvement. pratiquement du milieu pendant la période où les eaux sont froides et peu minérahisées (fig. 7). Cette disparition pourrait s'expliquer par une migration comportementale identique à celle observée par Pfannkuche (1980). En juin et août $25 \%$ environ des individus étaient matures. Selon Pfannkuche (op. cit.) cette espèce présente un cycle de développement biannuel avec une période de reproduction bien définie.

5 individus matures de $P$. riparius ont été récoltés à différentes périodes de l'année (l le 31/03, 3 le $10 / 06$ et 1 le $06 / 10$ ).

\section{Enchytraeidae}

Nous avons récolté 1 seul spécimen mature de $F$. callosa (st. 2 le $31 / 07$ ), de $F$. magna (st. 4 le $31 / 07$ ), de F. striata (stat. 8 le $27 / 11$ ) et de E. buchholzi (st. 10 le 05/04).

L'abondance de $C$. atrata présente peu de variations stationnelles. Cette espèce se reproduit tout au long de l'année; $55 \%$ des 346 individus capturés étaient matures. 10 individus matures de $F$. perrieri ont été récoltés le 25/01, 1 le $03 / 06$ et 1 le $30 / 09$; dans un torrent froid des Pyrénées, la période de maturité de cette espèce et de $C$. atrata est limitée à l'automne (Giani \& Lavandier 1977).

C. cognettii présente un maximum d'abondance à la fin de l'automne. Parmi les 177 individus récoltés, 13 étaient sexuellement développés ( 3 en octobre et 10 en décembre).

Près de $50 \%$ des $94 \mathrm{~F}$. bisetosa échantillonnées étaient matures ; la période de maturité s'étale sur toute l'année.

Dans la ria, $L$. brunoi est absente de la station 11 où la salinité est faible ; elle présente des pics d'abondance (fig. 7) en août aux stations 12 et 13 où la salinité at teint des taux de $6 \%$ et $7,5 \%$ respectivement ; les 62 individus matures récoltés ont été trouvés uniquement en été : 50 en août (49 à la st. 12) et 12 en juin ( 8 à la st. 13). Pendant la période, froide lorsque la salinité de la ria diminue fortement, L. brunoi semble disparaître complètement du milieu. Ces résultats, et la capture d'individus à tous les stades de développement - y compris des spécimens matures - suggèrent que dans la ria de Muros e Noia la période de développement de cette espèce serait extrêmement courte et qu'elle passerait la période hivernale sous la forme de cocons. 
Sur les 162 individus de $M$. argentea récoltés 25 étaient matures ( 1 le $27 / 01,1$ le 31/07, 2 le 30/09, 1 le $06 / 10$ et 20 le $27 / 11$ ). Le plus grand nombre de spécimens a été récolté a la fin de l'automne lors de la période de maturité.

Nous avons récolté 4 spécimens matures de $M$. riparia le 30/09 dans plusieurs stations d'eau courante et 21 de $M$. southerni au printemps et en été ( 1 le $01 / 04,19$ le 10/06 et 1 le 04/08).

\section{Densité, structure et diversité des peuplements}

Pour le traitement statistique des données, les Oligochètes non identifiables à l'état immature ont été répartis entre les diverses espèces du genre en fonction des pourcentages d'individus matures capturés. Bien que les résultats de nos récoltes dans les différentes zones de la lagune soient exprimés séparément, dans l'analyse nous les avons considérés globalement.

L'abondance relative de chaque espèce aux différentes stations est reportée dans le tableau II. Les figures 8 et 9 visualisent graphiquement, et de façon comparée, la densité, la structure et la diversité des peuplements.

Dans le secteur d'eau courante, la densité du peuplement est comparable à celle d'autres petites rivières non polluées (Armitage 1976, Dumnicka 1978, 1982). Elle augmente progressivement de l'amont vers l'aval (de $1350 \mathrm{ind} / \mathrm{m}^{2}$ à la st. 2 à $9308 \mathrm{ind} / \mathrm{m}^{2}$ à la st. 9). Seules les stations $7\left(9617\right.$ ind $\left./ \mathrm{m}^{2}\right)$ et 8 $\left(2866 \mathrm{ind} / \mathrm{m}^{2}\right)$ s'individualisent : la première présente un fond stable car elle est située en plaine juste après la confluence avec un important affluent ; la deuxième se trouve $50 \mathrm{~m}$ en aval d'une cascadelle, dans une zone à forte pente où le courant empêche l'installation de macrophytes et provoque une grande instabilité du milieu. La densité moyenne de peuplement du lac de barrage est d'environ $9000 \mathrm{ind} / \mathrm{m}^{2}$ et elle est comparable à celles observées dans d'autres réservoirs oligotrophes d'Espagne (Martinez-Ansemil \& Prat 1984). Au niveau de la ria, la densité de peuplement est faible à la station $11 \mathrm{du}$ fait de fréquentes extractions de sable. La méthode d'échantillonnage utilisée ne nous permet pas de connaître la densité du peuplement de la lagune.
L'abondance de substrats minéraux grossiers et de macrophytes en courant rapide (st $2,3,4,5,6$, 8 ,9) entraîne la dominance de deux espèces oxyphiles, $S$. heringianus et/ou $N$. alpina. A ces espèces se joignent $S$. lacustris dans les zones à courant modéré (st. $2,3,5$ ) recouvertes par des macrophytes, $S$. parvus à la station 8 dont le lit est formé exclusivement par un substrat grossier et $T$. ignotus à la station 6 où l'on trouve d'importants dépôts de sable fin sur les berges.

$S$. lacustris est également dominante dans la lagune (st. 1) du fait de l'abondance de végétation palustre avec, à un degré moindre, $N$. communis et $N$. variabilis. Le fond argileux de la station 7 permet le développement d'une importante population de $S$. velutinus et $L$. hoffmeisteri. Cette dernière espèce domine également les peuplements des fonds vaseux, riches en matière organique, du barrage.

Le peuplement de la ria, très nettement différencié, est dominé par des espèces capables de supporter d'importantes fluctuations dans le degré de minéralisation des eaux : $L$. brunoi, $N$. elinguis et $A$. monospermathecus. L. brunoi est même absent de la station située le plus en amont alors qu'il représente environ $35 \%$ du peuplement des deux stations aux eaux les plus minéralisées. Ces trois espèces ainsi que $P$. litoralis, $A$. sannio et $M$. southerni typiquement euryhalines, n'ont été trouvées que dans la ria dont elles forment la quasi-totalité de la faune d'Oligochètes.

Dans les stations d'eau courante situées le plus en amont (st. 2, 3, 4), la grande diversité d'habitats permet le développement d'une communauté riche en espèces et assez équilibrée : 35 à 41 espèces par station dont aucune ne représente plus de $26 \%$ du peuplement ( $\sigma$ compris entre 4 et 6,3 ).

Les biotopes lotiques des cours moyen et inférieur (st. $5,6,8,9$ ) ont une diversité spécifique encore élevée ( 21 à 36 espèces par station) mais avec des peuplements hétérogènes : l'espèce dominante représente toujours plus de $40 \%$ du peuplement et l'écart-type des pourcentages est remarquable ( $\sigma$ compris entre 8 et 12,9$)$. La diversité est faible à la station 7 oủ nous avons trouvé uniquement 12 espèces et où $S$. velutinus et $L$. hoffmeisteri forment environ $60 \%$ du peuplement. Dans le lac de barrage (st. 10) le nombre d'espèces est assez élevé (23) mais une seule, $L$. hoffmeisteri, forme les trois quarts du peuplement. 


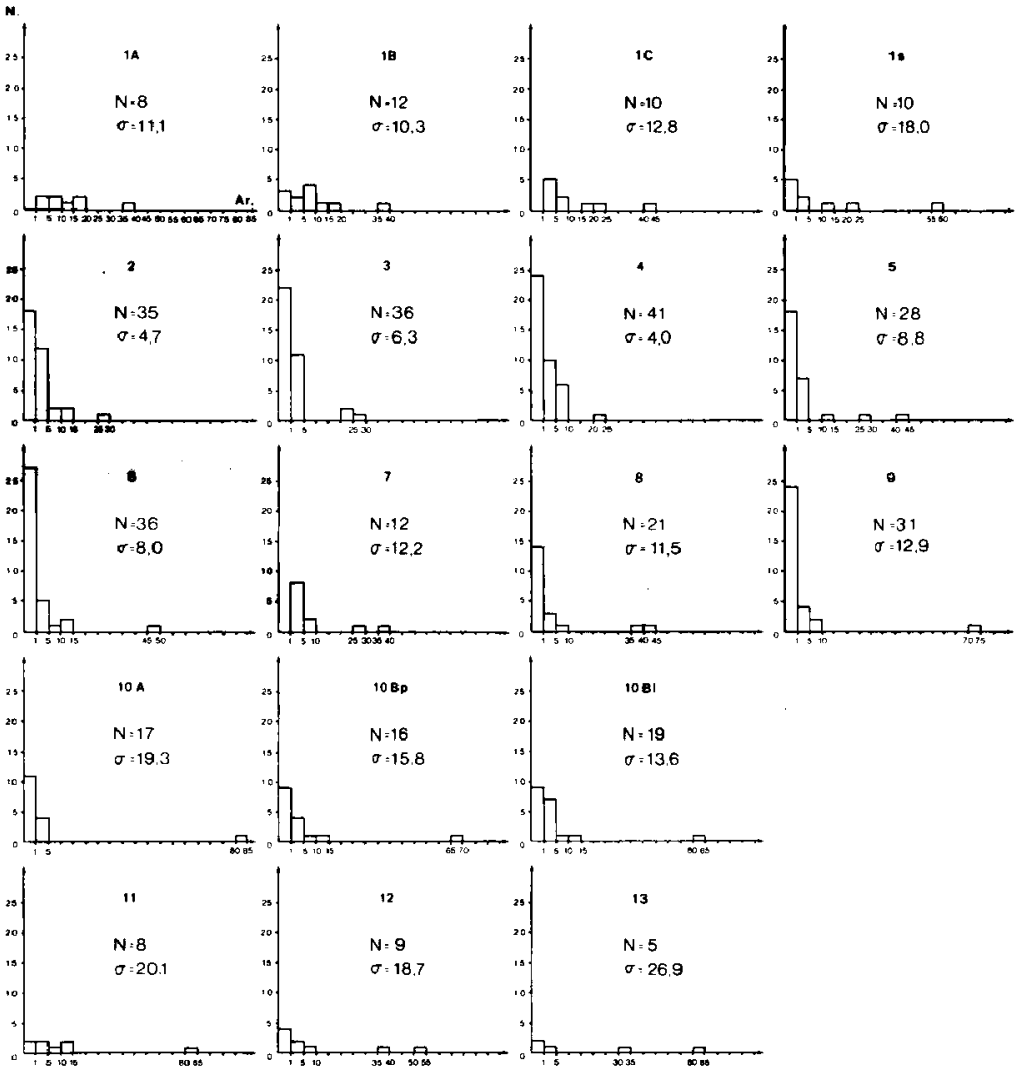

Fig. 8. Structure et diversité du peuplement. $\mathrm{N}$ : nombre d'espèces, Ar : abondance relative, $\sigma$ : écart type. 


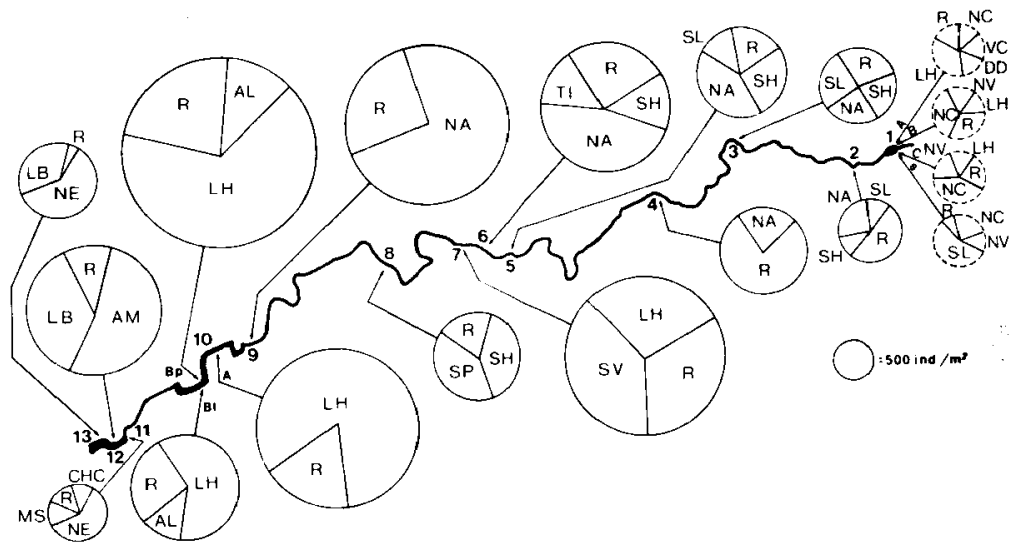

Fig. 9. Densité du peuplement et espèces dominantes (Ar $>10 \%$ ) aux différentes stations (pour la signification des symboles voir,fig. 10).

La diversité spécifique des trois stations de la ria est très faible ( 5 à 9 espèces) ; $N$. elinguis forme plus de $60 \%$ des effectifs à la station $11(\sigma=20,1), A$. monospermathecus avec $L$. brunoi près de $90 \%$ à la station $12(\sigma=18,7)$ et $N$. elinguis avec $L$. brunoi atteignent $95 \%$ à la station $13(\sigma=26,9)$.

\section{Répartition spatiale}

\subsection{Répartition longitudinale}

Compte tenu des différences de taux de reproduction des espèces d'Oligochètes nous n'avons pas utilisé directement les données brutes (abondance de l'espèce à la station). Nous avons accordé la même importance à toutes les espèces en répartissant leurs effectifs en 6 classes d'abondance (classes 0 à 5) ; la limite supérieure de la classe 5 correspond à l'abondance optimale c'est-à-dire au nombre d'individus récoltés dans la station où l'espèce a le plus fort effectif (A. max.). Les diffèrentes limites de classes ont été établies d'après la méthode de Verneaux (1973) qui considere par exemple la limite supérieure de la classe moyenne (classe 3 ) comme le rapport entre le nombre total d'individus capturés et le nombre de stations où l'espèce est présente $(\mathrm{Ai} / \mathrm{N})$.

Dans une première analyse incluant les 13 stations (fig. 10a) l'axe 1 explique $30,2 \%$ de la variance et l'axe 2 uniquement $13,6 \%$. La position relative sur l'axe 1 des stations de la ria traduit leur forte singularité faunistique par rapport à l'ensemble des stations d'eau douce. La projection du nuage de points sur l'axe 2 montre une séparation nette entre le milieu saumâtre, le milieu d'eau courante et le milieu d'eau stagnante ou à renouvellement lent et permet d'opposer la faune des milieux lotiques d'eau douce à celle qui se développe davantage ou exclusivement dans les milieux lénitiques. $N$. elinguis est abondante dans la ria alors qu'elle est totalement absente dans les stations d'eau douce. Bien que cette espèce ait été trouvée dans des eaux où la salinité atteint des valeurs de $25 \%$ (Lasserre 1966) elle est fréquemment signalée en eau douce ; malheureusement la plupart de ces citations ne mentionnent pas le degré de minéralisation de l'eau. L'abondance de cette espèce dans une rivière méditerranéenne fortement minéralisée (Giani \& Martinez-Ansemil 1981) et dans 
$A C=A$. camerani

AL = A. leydigi $i$

AH = A. mosospormathecus

AP $=A$. pluriseta

AS $=$ A. Sannio

BV = B. vejdovskyanum

CA $=c$. strata

$\mathrm{CC}=\mathrm{c}$. cognetii

$C D=c$. diastrophus

CG - C. glandulosa

CHC- $c$, erystallinus

$\mathrm{CHL}=\mathrm{c}$. langi

$\mathrm{CI}=$ c. Limnae

CS = C. sphagnetorum

DO = D. dorsalis

$88=E$. buchholri

$E T=E$. cetraedra

$\mathrm{FB}=\boldsymbol{F}$. bisecosa

$F C=F$, callosa

$\mathbf{F H}=F$, angra

$F P=F$, perrieri

FS $=F$. striate

HG $=H$. gordioides

HSP - Henlea sp.

CB $=$ L. brunOi

LH - L. hoffmeisteri

$\mathrm{LU}=L$. udekemianus

$L V=L$. variegatus

$M A=M$. argentea

MR = H. riparia

HS = H. southerni

MSP= Aesenchytraeus sp.

$\mathrm{NA}=\mathrm{N}$. Alpina

NB $=N$. bretscheri

$\mathrm{NC}=N$. commusis

NE $=N$. elinguis

$\mathrm{NV}=N$, variabilis

$P A=P$. amphibiotica

$P A E=P$, aeguiseta

$\mathrm{PAL}=P$. $a \angle b i c O L a$

$\mathrm{PB}=P$. bilobata

PJ - $P$. jenkinat

$\mathrm{PL}=\mathrm{P}$. Litoralis

$\mathrm{PM}=P$. menoni

$\mathrm{PN}=\mathrm{P}$. notopors

PO = P. osbarni

$\mathrm{PR}=$ P. riparius

PS = P. Sima

$P T=P$. tourengui

$\mathrm{PV}=P . v 01 \mathrm{k} i$

$R C=R$. coccineus

$\mathbf{R F}=R$. falciformis

RS $=R$, subterraneus

SA $=S$. appendiculata

$S B=S$. brachystilus

$\mathrm{SH}=s$. heringianus

$\mathrm{SJ}=s$. josinae

SL = s. lacustris

$S P=S$. paruug

$S V=s$. velutinus

TGS= Tubificidae g. sp,

$\mathrm{TI}=\mathrm{r}$. ignotus

TSP = Trichocrilus sp.

$T T=T$. tubifex

UU $=0$. uncinata

$v c=v$. comata


Fig. 10. Groupement des stations en fonction des peuplements d'Oligochètes. Analyse portant sur les 13 stations. 
les stations de la ria, son absence des stations d'eaux douces du Tambre et quelques données de la littérature (Korn 1963, Ladle 1971, Lafont \& Juget 1976, Lafont 1977, Ródriguez 1984) prouvent l'affinité de cette espèce pour les eaux fortement minéralisées. $N$. elinguis se développe aussi davantage à la faveur d'un enrichissement des eaux en matière organique (Lafont \& Juget 1976, Lafont 1977, Giani 1984).

Les trois ensembles de stations séparés dans cette première analyse masquent totalement l'évolution du peuplement d'Oligochètes le long du cours axial du Tambre. Une deuxième analyse (Fig. 10b) portant uniquement sur les stations typiques du cours d'eau (st. $2,3,4,5,6,8,9$ ) a été effectuée.

Les deux premiers axes expliquent $59,8 \%$ de la variance $(43,8 \%$ et $16 \%)$. Le premier facteur $n$ 'a pas de signification écologique véritable : la projection des points-espèces sur l'axe 1 reflète uniquement un gradient d'occupation des classes d'abondance. L'axe 2 traduit la distribution des espèces entre les différentes stations qui se succèdent en fonction de leur localisation sur le cours d'eau selon un axe amont-aval.

La majorité des espèces d'Enchytraeidae semblent trouver les conditions les plus favorables pour leur développernent dans le cours supérieur (st. 2, 3, 4) ; seules $C$, atrata et $M$. argentea sont également bien représentées dans les cours moyen et inférieur (st. $5,6,8,9)$.

$H$. gordioides et la majorité des Tubificidae se développent davantage dans les cours moyen et inférieur mais la plupart des Rhyacodrilinae $(R$. falciformis, $R$. coccineus et $P$. tourenqui) sont confines dans le cours supérieur où ils forment des populations relativement importantes.

Les Lumbriculidae sont bien représentés tout le long du cours d'eau sauf $S$. brachystilus qui se cantonne dans le cours supérieur.

Les Naididae, considérés dans leur ensemble, ne montrent pas de distribution bien définie le long du cours d'eau ; la plupart des espèces ont une large répartition longitudinale.

La distribution des Oligochètes le long du Tambre ne présente pas une véritable zonation au sens d'Illies \& Botosaneanu (1963). Il existe cependant une différence entre le cours supérieur (st. 2, 3, 4) et les cours moyen et inférieur ; cette différence résulte du plus grand développement de la plupart des espèces d'Enchytraeidae et de certaines espèces de Lumbriculidae ( $S$. brachystylus) et de Rhyacodrilinae ( $R$. falciformis, $R$. coccineus et $P$. touren$q u i)$ dans le cours supérieur.

Ces observations combinées avec celles de Wachs (1967), Kasprzak \& Szczesny (1976), Giani \& Lavandier (1977), Learner et al (1978), Giani \& MartinezAnsemil (1981), Dumnicka (1982) et Schwank (1982a) montrent que les Enchytraeidac, les Naididae et les Lumbriculidae sont les familles d'Oligochètes les mieux représentées dans le rithron (les Enchytraeidae dominant dans le cours supérieur et les Naididae dans le cours moyen) alors que les Tubificidae se développent davantage dans le cours inférieur (potamon). L'abondance de Rhyacodrilinae dans le cours supérieur du Tambre ne va pas à l'encontre de ces observations car le terme Tubificidae fait ici référence aux formes pélophiles (essentiellement Tubifex et Limnodrilus). La présence du barrage de Barrié de la Maza sur le cours inférieur du Tambre exclue l'existence d'un véritable potamon.

\subsection{Répartition horizontale}

Au niveau d'une station donnée, la composition chimique et la température de l'eau sont pratiquement homogènes. Le substrat (au sens large : support, abri, nourriture) et le courant sont les principaux facteurs responsables de la distribution des différentes populations au sein d'une même station (répartition horizontale).

Nous avons analysé l'influence du courant et du substrat au moyen d'une analyse factorielle du même type que la précédente mais portant uniquement sur les espèces représentées par un nombre d'individus $\geqslant 100$ (Tableau III) ; les données ont été regroupées en quatre classes d'abondance (classes $0,1,2$, 3).

Les axes 1 et 2 (fig. 11 ) expliquent $61 \%$ de la variance (espèces et biotopes) et l'axe 3 seulement $8 \%$.

Bien que la vitesse du courant et le substrat soient deux facteurs fortement liés, c'est le courant qui préside à la distribution des variables-espèces et des variables-biotopes en rapport avec l'axe $1(37,3 \%$ d'inertie). En effet, les biotopes où la vitesse du courant est importante (très rapide à modérée) se situent, à quelques rares exceptions près, du côté négatif de cet axe alors que ceux dont le courant est très lent ou nul se placent du côté positif. La position 


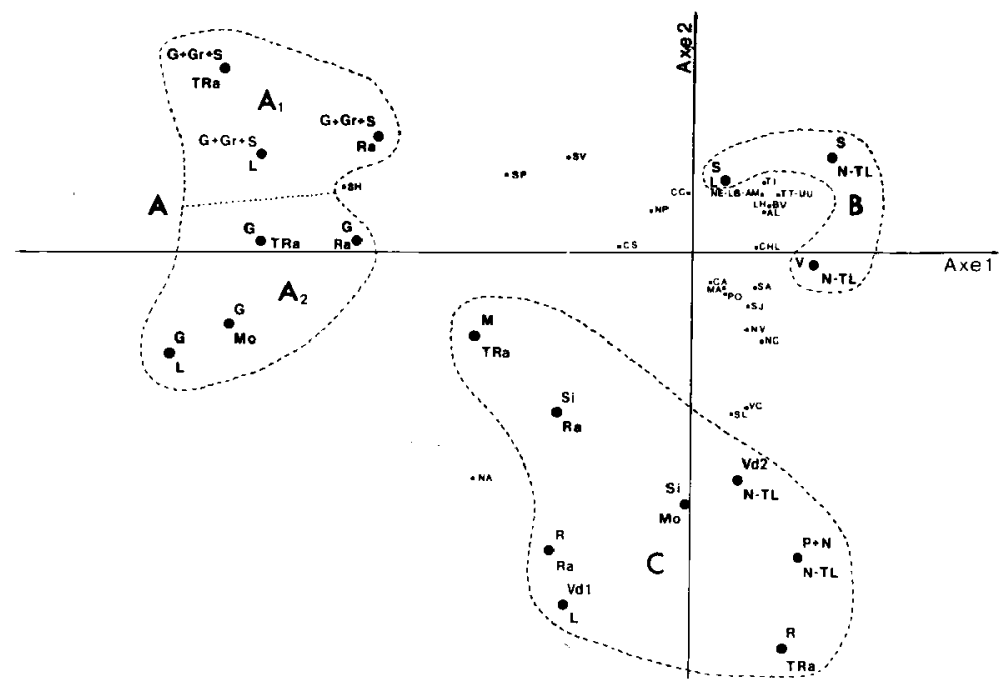

Fig. 11. Répartition longitudinale des Oligochètes dans le cours d'eau. Analyse factorielle des correspondances restreinte aux stations du milieu lotique.

intermédiaire des fonds de macrophytes en courant rapide ou même très rapide peut être expliquée par l'abri que cette végétation offre aux Oligochètes.

Le deuxième facteur de répartition au niveau stationnel $(23,7 \%$ d'inertie) marque la différence entre les substrats minéraux (côté positif et début du côté négatif de l'axe 2) et ceux recouverts par des macrophytes ou des débris végétaux (extrême négatif).

Dans le plan défini par ces deux facteurs on peut délimiter trois ensembles de biotopes :

- Ensemble A : fonds de galets accompagnés ou non par des éléments plus fins (gravier, sable) en courant modéré, rapide ou très rapide. Dans cet ensemble nous pouvons délimiter deux sousensembles ; le premier (Al) est constitué par des éléments de granulométrie variée alors que le second (A2) regroupe uniquement des substrats minéraux grossiers (galets).

- Ensembre B : substrats sablonneux ou vaseux en courant lent, très lent ou nul avec un certain dépôt de matière organique.

- Ensemble C : biotopes caractérisés par la présence de macrophytes vivantes ou de débris végétaux.

$S$. heringianus et $S$. parvus sont les espèces qui contribuent le plus à l'isolement de l'ensemble A ; $S$, heringianus est très souvent l'espèce dominante dans les substrats minéraux en faciès lotique avec des densités de population qui peuvent atteindre $7800 \mathrm{ind} / \mathrm{m}^{2}$. Le caractère pétricole et réophile de cet te espèce a déjà été signalé (Wachs 1967, Timm 1970, Ladle 1971, Giani 1976, Giani \& Lavandier 
1977. Ródriguez 1984) ; les références relatives à l'habitat de $S$. parvus sont beaucoup plus rares mais corroborent nos données (Juget 1959, 1979, Giani \& Martinez-Ansemil 1981, Ródriguez 1984). S. veluunus contribue aussi à la différenciation de cet ensemble et plus particulièrement du sous-ensemble Al car elle nécessite la présence d'éléments minéraux fins pour son développement. Cette espèce semble confinée aux lacs profonds et aux petites rivières bien oxygénées avec des substrats minéraux comportant des éléments fins (Juget 1958, Brinkhurst 1964, Giani 1976, Juget \& Lafont 1979).

Les espèces qui participent de façon décisive à la différenciation de l'ensemble B sont : $A$. monospermathecus, L. brunoi, $N$. elinguis, $T$. ignotus, $T$. tubifex, L. hoffmeisteri, U. uncinata, B. vejdovskyanum et $A$. leydigii. Le caractère psammophile d' $A$. monospermathecus, $L$. brunoi et $N$. elinguis résulte de leur seule présence dans la ria. $N$. elinguis est cependant commune dans les eaux douces.

$T$. ignotus n'a été trouvée que dans les fonds sablonneux du secteur d'eau courante notamment dans le sable fin ; $A$. leydigii n'a pratiquement été récoltée que dans la vase de la zone profonde du barrage ; les quatre autres espèces de cet ensemble peuplent les fonds sablonneux ou vaseux du secteur d'eala courante en facies lénitique et/ou des biotopes d'eau stagnante. Ces résultats sont en accord avec les données de la littérature.

Les espèces les plus représentatives de l'ensemble C sont : S. lacustris, V. comata et $N$. alpina. $S$. lacusris est un habitant typique des milieux stagnants où sa présence est généralement associée au développement des macrophytes ; bien que dans le Tambre cette espèce se développe davantage parmi la végétation palustre de la lagune, elle forme aussi des populations denses parmi les macrophytes en courant. Le caractère phytophile de cette espèce est bien connu (Berg 1938, 1948, Laakso 1969b, Timm 1970, Schwank 1982b).

$V$. comata est une espece caractéristique des tourbières (Piguet \& Bretscher 1913, Timm 1970, Juget \& Giani 1974) ; nous l'avons récoltée surtout parmi la végétation palustre de la lagune et dans les amas de débris végétaux en état avancé de décomposition sur les berges des stations d'eau courante ; elle est également abondante parmi les macrophytes en courant.
Contrairement aux deux espèces précédentes, $N$. alpina est caractéristique des milieux lotiques (Wachs 1967, Giani 1976, Lafont 1977, Giani \& MartinezAnsemil 1981, Dumnicka 1982, Schwank 1982b). Elle est absente de la lagune mais forme des populations très denses parmi les macrophytes en courant où elle est souvent l'espèce dominante; un nombre important d'individus a été récolté sur les substrats minéraux grossiers en courant et parmi les débris végétaux retenus sur les berges des stations à forte circulation d'eau. Selon Wachs (1967) et Schwank (1982b) N. alpina colonise de préférence les substrats minéraux en courant. Plus que le substrat, le caractère réophile de cette espèce à la recherche d'eaux bien oxygénées semble être le critère essentie! qui régit sa distribution dans les cours d'eau.

Toutes les autres espèces considérées dans cette analyse présentent une amplitude d'habitat supérieure vis-à-vis du substrat et/ou du courant et de ce fait participent de façon négative à la différentiation des ensembles de biotopes.

\subsection{Répartition dans le lac de barrage}

La répartition bathymétrique de la faune d'Oligochètes du lac de barrage de Barrié de la Maza (st. 10) ne présente pas le profil classique des milieux stagnants profonds (Tableau II ; figs 8 et 9). L'absence d'une véritable stratification thermique, la sursaturation en $\mathrm{O}_{2}$ des eaux du fond, l'instabilité de la zone littorale, conséquence du marnage et l'absence de végétation, sont à l'origine d'une certaine inversion des résultats. Ainsi, la richesse spécifique globale et la diversité de Naididae atteignent des valeurs aussi importantes dans la zone profonde que dans la zone littorale et la densité de l'ensemble du peuplement et l'abondance de Naididae sont même nettement plus élevées dans la première. L'abondance de débris végétaux allochtones dans la zone littorale et la présence dans la zone profonde de sédiments fins au niveau du secteur B et grossiers dans le secteur A semblent jouer un rôle important dans la distribution de la faune.

\section{Discussion}

Parmi les différents aspects abordés dans cette étude les facteurs de répartition retiennent plus particulièrement l'attention car les données concernant des cours d'eau non pollués sont rares et souvent fragmentaires. Lorsqu'un cours d'eau est soumis à 
Tableau II. Abondance relative des diverses espèces d'Oligochetes et nombre total d'Oligochetes récoltés à chacune des stations. $s$ : surface : $p$ : zone profonde $; 1$ : zone littorale $; i$ : individus immatures.




Tableau III. Préférendums des Oligochètes vis-à-vis du substrat et de la vitesse du courant.

0 : présence pour les espèces à faible effectif ( $\geqslant 100$ individus) ; $, * *, * *$ : abondance des espèces à fort effectif ( $\geqslant 100$ individus).

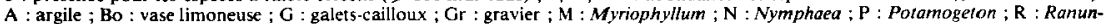
cuius ; $\mathrm{S}:$ sable : $\mathrm{Si}:$ Sium ; $\mathrm{V}$ : vase riche en matière organique ; Vdl et Vd2 : débris végétaux dans un état de décomposition peu ou très avancé.

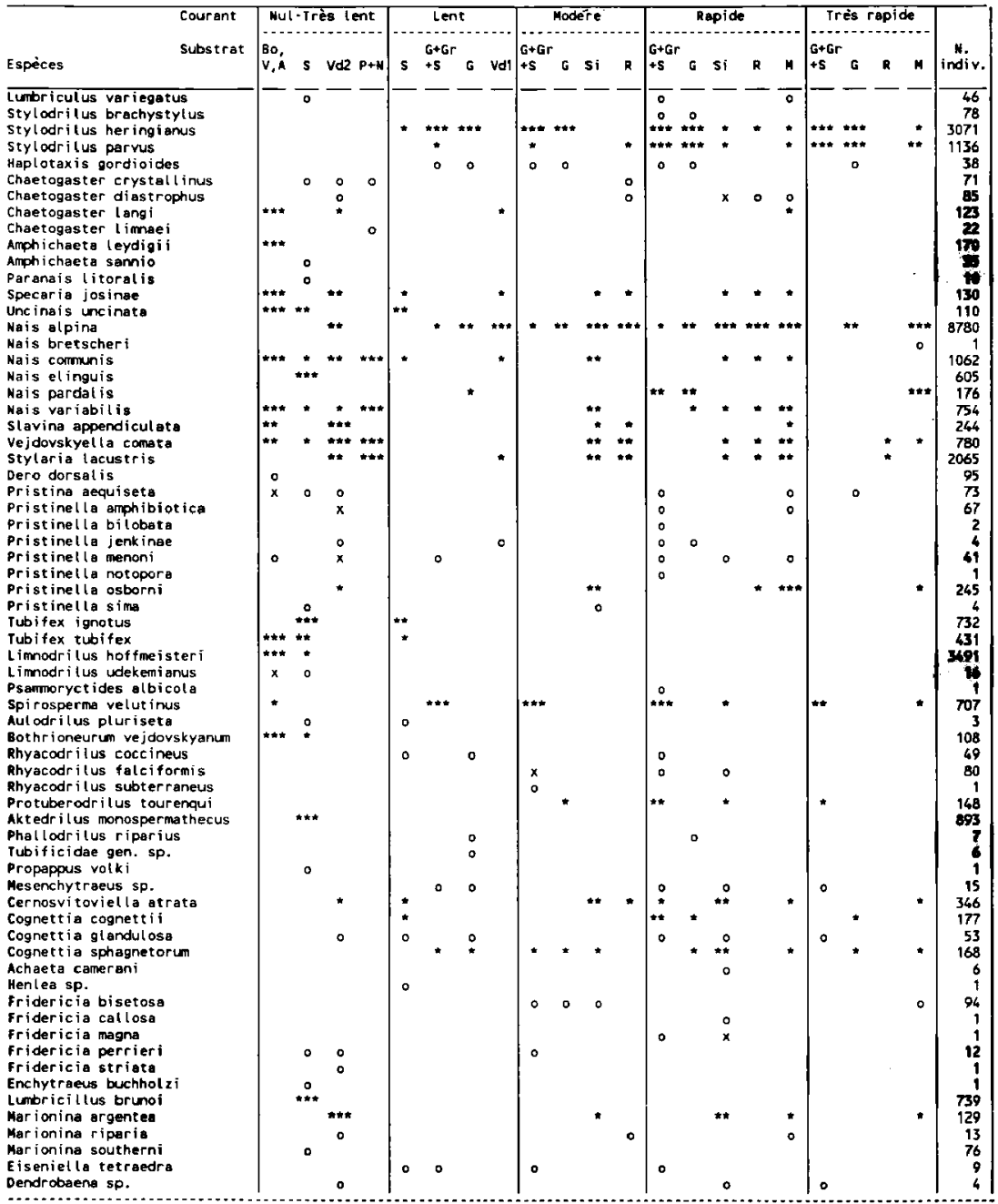


une pollution organique intense celle-ci devient un des facteurs qui déterminent la composition et la répartition de sa faune d'Oligochètes (Dumnicka 1978, Lafont 1977, Uzunov 1982). Cependant, selon Wachs (1967) et Kasprzak \& Szczesny (1976), en l'absence d'une véritable pollution les principaux facteurs responsables de la répartition des populations d'Oligochètes le long d'un cours d'eau sont le substrat et le courant. Dans de telles conditions, Wachs (op. cit.) note l'indépendance des Oligochètes vis-à-vis de la composition chimique des eaux alors que Kasprzak \& Szczesny (op. cit.) soulignent leur quasi-totale indépendance vis-à-vis de la température. Seules de fortes modifications de ces facteurs peuvent casser le profil longitudinal de la répartition de la faune d'Oligochètes comme nous l'avons déjà constaté : brusque augmentation de la minéralisation des eaux dans la rivière Argens après sa confluence avec un affluent salé (Giani \& Martinez-Ansemil 1981). Le rôle prédominant du substrat et du courant dans la répartition des Oligochètes ont été soulignés aussi par Korn (1963), Learner et al. (1978), Dumnicka (1978, 1982), Giani \& Martinez-Ansemil (op. cil.), Schwank (1982a), Munoz et al. (1986).

La composition chimique des eaux du Tambre, qui varie peu le long du cours, ne semble pas influencer la repartition longitudinale des Oligochètes. On pourrait supposer que la température joue un rôle en raison de la forte amplitude thermique des eaux du Tambre entre l'amont et l'aval mais, même des espèces souvent considérées comme sténothermes d'eau froide tels $N$. alpina (Wachs 1967, Giani 1976, Learner et al. 1978, Schwank 1982a) ou $S$. velutinus (Brinkhurst 1964, Giani 1976, Juget \& Lafont 1979) sont bien représentées dans la station d'eau courante la plus aval (st. 9) où la température de $l^{\prime}$ 'eau varie entre 6,5 et $20,6^{\circ} \mathrm{C}$ en cours d'année.

Dans les milieux naturels, comme le Tambre, ce serait l'alternance de mosaïques de biotopes déterminées par des différences dans la nature et la composition du substrat et la vitesse du courant - biotopes de $3^{c}$ ordre d'Albrecht (1953) - qui seraient responsables des changements longitudinaux de la composition et de la structure du peuplement d'Oligochètes. Comme nous l'avons vu, une grande partie des espèces sont eurytopes ce qui entraîne un important chevauchement des populations ; lestendances observées dans la distribution résultent du fait que les espèces les plus abondantes ( $S$. heringianus, S. parvus, N. alpina, S. lacustris et $T$. ignotus) ont un préférendum assez défini vis-à-vis du substrat et de la vitesse du courant.

En ce qui concerne la période de maturité des différentes espèces et l'évolution saisonnière des populations, quatre aspects essentiels ressortent de cette étude :

1) Dans le Tambre la plupart des espèces de Naididae n'ont été trouvées qu'à l'état immature : les récoltes d'individus sexuellement développés ne représentent qu'un faible pourcentage du peuplement à la période considérée. $A$. leydigii constitue la seule exception ; cette espèce récoltée uniquement dans le lac de barrage, semble se reproduire massivement par voje sexuée juste avant l'arrivée d'ıne période défavorable à son développement dans ce milieu. L'apparition de naididés matures dans le Tambre coincide généralcment avec l'augmentation appréciable de la température de l'eau à la fin du printemps et au début de l'été.

2) Dans le secteur d'eau douce du Tambre la plupart des espèces abondantes de Lumbriculidae, Tubificidae et Enchytraeidae sont matures tout le long de l'année.

3) Toutes les espèces présentes uniquement dans la ria ( $N$. elinguis, $A$. monospermathecus, $L$. brunoi et $M$. southerni) ont une période de maturité limitée à la saison chaude (fin du printemps-été).

4) D’une manière générale, les périodes les plus favorables pour la reproduction asexuée des Oligochètes du Tambre sont le début du printemps, l'hiver et surtout le début de l'automne. L'été est la saison la plus défavorable pour la scissiparite ; $C$. limnaei, espèce capturée uniquement à la lagune, constitue à cet égard la seule exception mais son cycle de développement pourrait être directement en relation avec le cycle de développement des mollusques hôtes (Gruffydd 1956, Streit 1974).

Nos résultats confirment ou élargissent la gamme des divers cycles de développement des Oligochètes en milieu naturel. De véritables progrès dans la connaissance de ce cycle ne pourront guère provenir que de travaux expérimentaux approfondis ou d'études minutieuses sur le terrain ; seul un échantillonnage intensif permettra d'enregistrer les véritables explosions de populations qui peuvent se produire en l'espace de quelques jours (Lochhead \& Learner, 1983), d'appréhender des passages rapides 
à l'état de maturité d'une partie importante des effectifs (Loden 1981) et de suivre l'évolution d'un grand nombre de facteurs abiotiques (ex. variation ponctuelle du gradient thermique) mais aussi biotiques (ex. explosions dans la production primaire, brusque augmentation de la pression de prédation).

D'une manière générale les cycles de développement des Oligochètes qui peuplent le secteur d'eau courante du Tambre sont caractéristiques d'un milieu plutôt stable et assez productif (importants apports de matière allochtone provenant de l'abondante végétation riveraine) ; les principaux traits différentiels sont le faible nombre de Naididae matures, une période de maturité étalée sur toute ou la plus grande partie de l'année pour les autres familles et une distribution assez homogène des effectifs des populations pendant l'année. Au contraire, le milieu saumâtre de la ria se caractérise par son instabilité qui induit des cycles de développement mieux définis et plus tranchés. Le lac de barrage représente, à cet égard, une situation intermédiaire.

\section{Remerciements}

Je remercie N. Giani et J.N. Tourenq du Laboratoire d'Hydrobiologie de l'Université Paul Sabatier de Toulouse pour leur appui scientifique et leurs encouragements lors de la réalisation de ce travail.

Une partie de cette étude a été réalisée dans le cadre d'un programme financé par la C.A.I.C.Y.T. (P.B. 86-0406).

\section{Travaux cités}

Albrecht (M.L.). 1953. - Die Plane und andere Flämingbache (Ein Beitrag zur Kenntniss der Fließgewässer der Norddeutschen Tiefebene). Z. Fish Hilfswis, $1: 390-473$.

Armitage (P.D.). 1976. - A quantitative study of the invertebrate fauna of the River Tees below Cow Green Reserfoir. Freshwat. Biol., 6 : 229-240.

Berg (K.). 1938. - Studies on the bottom animals of Esrom Lake. $K$. danske Vidensk. Selsk. Skr,, $9(8): 1-255$.

Berg (K.). 1948. - Biological studies on the River Susaa. Folio Limnol. Scand., $4: 1-318$.

Brinkhurst (R.O.). 1964. - Observations on the biology of lakedwelling Tubificidae. Arch. Hydrobiol., 60 (4) : 385-418.

Brinkhurst (R.O.) \& Kennedy (C.R.). 1965. - Studies on the biology of the Tubificidae (Annelida, Oligochata) in a polluted stream. J. Anim. Ecol, 34 : 429-443.

Carballeira (A.), Devesa (C.), Retuerto (R.), Santillan (E.) \& Ucieda (F.). 1983. - Bioclimatología de Galicia. Fundación Barrié de la Maza, A Coruña : 391 p.

Cernosvitov (L.). 1928. - Die Oligochaeten fauna der Karpathen. Zool. Jb. Syst., $55: 1-28$.

Dumnicka (E.). 1978. - Communities of Oligochaetes (Oligochaeta) of the River Nida and its tributaries. Acta Hydro. biol., 20 (2) : 117-141.
Dumnicka (E.). 1982. - Stream ecosystems in mountain grassland (West Carpathians), 9. Oligochaeta. Acta Hydrobiol., 24 (4) : 391-398.

Eyres (J.P.), Williams (N.V.) \& Pugh-Thomas (M.). 1978. Ecological st udies on Oligochacta inhabiting depositing substrata in the Irwell, a polluted English river. Freswat. Biol., $8: 25-32$.

Frenzel (P.). 1981. - Untersuchungen zur Okologie der Naididae des Bodensees. Die Nische von Chaetogaster und Amphichaela. Arch. Hydrobiol., 91 (1) : 45-55.

Giani (N.). 1976. - Les Oligocthètes aquatiques du Sud-Ouest de la France. Anm/s Limnol., 12 (2) : 107-125

Giani (N.). 1984. - Le Riou Mort, affluent du Lot, pollué par métaux lourds. IV. Etude des Oligochètes. Annis Limnol., $20(3): 167-181$.

Giani (N.) \& Lavandier (P.). 1977. - Les Oligochètes du torrent d'Estaragne (Pyrénées Centrales). Bull. Soc. Hist. Nat. Toulouse. 113 (1-2) : 234-243.

Giani (N.) \& Martinez-Ansemil (E.). 1981, - Contribution à là connaissance des Oligochètes aquatiques du bassin de l'Argens (Var, France). Annis Limnol., 17 (2) : $121-141$.

Gruffydd (L.D.) 1956. - The population biology of Chaetogaster limnaei limnaei and Chaetogaster limnaei vaghini (Oligochaeta). J. Anim. Ecol., $34: 667-690$.

Illies (J.) \& Botosaneanu (L.). 1963. - Problèmes et méthodes de la classification et la zonation écologique des eaux courantes, considérées surtout du point de vue faunistique. Mitt. Int. Verein. Theor. Angew. Limnol, $12: 1.57$.

Inst. Invest. Geol. Edaf. y Agrobiol. de Galicia. 1967. - Estudio agrobiológico de la provincia de La Coruña. Pub. Diputac. Provinc. La Coruña : $349 \mathrm{p}$.

Juget (J.). 1958. - Recherches sur la faune de fond du Léman et du lac d'Annecy. Ann. Stat. centr. Hydrobiol. appl., 7 : 7-95.

Juget (J.). 1959. - Recherches sur la faune aquatique de deux grottes du Jura méridional français : la grotte de la Balme (Isère) et la grotte de Corveissiat (Ain). Ann. Speleol., 14 : $391-401$.

Juget (J.). 1967, - Quelques données nouvelles sur les Oligochètes du Léman : composition et origine du peuplement. Ann's Limnol., 3 (2) : 217-229.

Juget (J.) . 1979. - La texture granulométrique des sédiments et le régime alimentaire des Oligochètes limicoles. Hydrobiologia, 65 (2) : 145-154.

Juget (J.) \& Giani (N.). 1974. - Répartition des Oligochètes lacustres dans le Massif de Ncouvielle (Hautes-Pyrénées) avec la description de Peloscolex pyrenaicus n. sp. Annls Limnol., 10 (1): 33-53.

Juget (J.) \& Lafont (M.). 1979. - Description de Peloscolex furquini, n. sp. et redescription de Peloscolex moszynskii, Kasprzak, 1971 (Tubificidae, Oligochaeta), avec quelques remarques sur la répartition du genre Peloscolex dans les eaux douces frarç̧aises. Bull. mens. Soc. linn. Lyon, $2: 75-80$ et 113-118.

Kasprzak (K.). 1973, - Notatki o faunie skaposzczetow (Oligochaeta) Polski, I. Fragmenta faun., 18 (21) : 405-434.

Kasprzak (K.) \& Szczesny (B.). 1976. - Oligochaetes (Oligochaeta) of the River Raba. Acta Hydrobiol, 18 (1) : 75-87.

Kennedy (C.R.). 1966. - The life history of Limnodrilus hoffmeisteri Clap. (Oligochaeta : Tubificidae) and its adaptative significance. Oikos, $17: 158-168$. 
Korn (H.). 1963. - - Studien zur Ökologie der Oligochaeten in der oberen Donau unter Berücksichtigung der Abwassereinwirkungen. Arch. Hydrobiol., 27 (2) : 131 -182.

Laakso (M.). 199a. - New records of aquatic Oligochaeta from Finland. Ann. Zool. Fenn., $6: 348-352$.

Lakso (M.). 1969b. - Oligochaeta from brackish water near Tvärminne, southwest Finland. Ann. Zool. Fenn., $6: 98111$.

Ladle (M.). 1971. - The biology of Oligochaeta from Dorset chalk streams. Freshwat. Biol., 1 : 83-97.

Lafont (M.). 1977. - Les Oligochètes d'un cours d'eau montagnard pollué : le Bief Rouge, Annls Limnol., 13 (2) : 157-167.

Lafont (M.) \& Juget (J.). 1976. - Les Oligochètes du Rhône. I. Relevés faunistiques généraux. Ann's Limnol., 12 (3) : 253-268.

Lage-Yusty (M.A.). 1979. - Estudio de las aguas del rio Tambre. Thèse Univ. Santiago: $175 \mathrm{p}$.

Lasserre (P.). 1966. - Oligochètes marins des côtes de France. I. Bassin d'Arcachon : systématique. Cah. Biot. Marine, 7 : 295-317.

Learner (M.A.), Lochhead (G.) \& Hughes (B.D.). 1978. - A review of the biology of British Naididae (Oligochaeta) with emphasis on the lotic environment. Freshwat. Biol., 8 : 357-375.

Loden (M.S.). 1981 - Reproductive ecology of Naididac (Oligochaeta). Hydrobiologia, $83: 115-123$.

Loden (M.S.) \& Harman (W.J.). 1980. - Ecophenotipic variation in setae of Naididae (Oligochaeta). In Aquatic Oligochaete Biology. R.O. Brinkhurst \& D.G. Cook (eds.), Plenum Press, New York : 33-39.

Martinez-Ansemil (E.) \& Giani (N.). 1982. - Contribución al conocimiento del genero Pristina (Oligochaeta, Naididae) en Ia Peninsula lbérica. Bol. R. Soc. esp. Hist. nat. (Biol.), 80 (3-4) : 249-260.

Martinez-Ansemil (E.) \& Prat (N.). 1984. - Oligochaeta from profundal zones of Spanish reservoirs. Hydrobiologia, 115 : 223-230.

McMurray (S.), Vincent (B.) \& Vaillancourt (G.). 1982. - Variations saisonnières de la température de l'eau et cycle de développement de Limnodrilus hoffmeisteri Claparède (Oligochaeta : Tubificidae). Noturaliste can., $109: 223-228$.

Membiela-Iglesia (P.) \& Martinez-Ansemil (E.). 1984. - Larvas de Plecópteros del rio Tambre (Galicia) : estudio ecológico. Bol. Asoc. esp. Entom. $8: 101-109$.

Muñoz (I.), Prat (N.), Millet (X.) \& Martinez-Ansernil (E.). 1986. - Heterogeneidad espacial en la disträbución de los macroinvertebrados a lo largo de un transecto en el rio Llobregat (Barcelona, España). Limnetica, 2 : 135-145.

Pfannkuche (O.). 1980. - Distribution, abundance and life cycles of Oligochaeta from the marine hygropsammal with special reference to the Phallodrilinae (Tubificidae). Int. Rev. ges. Hydrobiol, 65 (6) : 835-848.

Pfannkuche (O.). 1981. - Distribution, abundance and life cycles of aquatic Oligochaeta (Annelida) in a freshwater tidal flat of the Elbe Estuary. Arch. Hydrobiol., 43 (4) : 506-524.

Piguet (E.). 1906. - Observations sur les Naididées et révision systématique de quelques espèces de cette famille. Revue Suisse Zool., 14 : 185-315.

Piguet (E.) \& Bretscher (K.). 1913. - Oligochètes. Catalogue des invertébrès de la Suisse. Mus. Hist. nat. Genève, 7 : 1-214.
Poddubnaya (T.L.). 1980. - Life cycles of mass species of Tubificidae (Oligochaeta). In Aquatic Oligochaete Biology. R.O. Brinkhurst \& D.G. Cook (eds.), Plenum Press, New York : 175-184.

Ródriguez (P.). 1984. - Los Oligoquetos acuáticos del río Nervión (Vizcaya España) = resultados faunisticos generalcs. Limnerica, 1 : 169-178.

Schwank (P.). 1982a. - Turbellarien, Oligochacten und Archianneliden des Breitenbachs und anderer oberhessischer Mittelgebirgsbäche. IV. Allgemeine Grundlager der Verbreitung von Turbellarien und Oligochaeten in Fliessgewässern. Arch. Hydrobiol, 62 (2) : 254-290.

Schwank (P.). 1982b. - Turbellarien, Oligochaeten und Archianneliden des Breitenbachs und anderer oberhessischer Mittelgebirgsbäche. III. Die Taxozönosen der Turbellarien und Oligochaeten in Fliessgewässern eine synökologische GliederungArch. Hydrobiol., 62 (2) : 191-253.

Smith (N.E.). 1985a. - Population and reproductive dynamics of Nois communis (Oligochaeta : Naididae) from a Wisconsin limnocrene. Am. Midt. Nat, , 114 (1): 152-158.

Smith (N.E.). 1985b. - Setal morphology and is intraspecific variation in Dero digitata and Dero nivea (Oligochaeta : Naididae). Trans. Am. Microsc. Soc., 104 (1): 45-51.

Smith (N.E.). 1986. - Ecology of Naididae (Oligochaeta) from an alkaline bog stream : life history patterns and community structure. Hydrobiologia, $133: 79-90$

Streit (B.). 1974. - Populationsdynamik von Chaetogaster limnaei limnaei in einer Population von Ancylus fuviatilis. Arch. Hydrobiol., 47 (1): 106.118.

Thomas (A.G.B.), Giani (N.) \& Thomas (N.), 1979. - Actions humaines sur la faune benthique torrenticole dans le SudOuest de la France. I. La Rigole de la Montagne Noire. Bull. Soc. Hist. nat. Toulouse, $115: 30-52$.

Timm (T.). 1967. - The life cycle of some Oligochaeta. Trudy Karel'skogo Ordeleniya Gosniorkh, 5 (1) : 202-204. (Traduction du russe).

Timm (T.). 1970. - On the fauna of the Estonian Oligochaeta. Pedobiologia, 10 (1) : 52-78.

Timm (T.). 1979. - The Estonian Lumbriculidae. Eesti Nsv Teaduste Akad. Toimitised, 28 (3): 158-171.

Uzunov (J.). 1982. - Statistical assessment of the significance of both bottom substrata and saprobity for the distribution of aquatic Oligochaeta in rivers. Limnologica (Berl.), 14 (2) : 353-361.

Varela-Allegue (R.). 1978. - Aportaciones al estudio floristico y ecológico de la laguna de Sabrado de los Monjes (La Coruña). Tesis de licenc. Univ. Santiago : $142 \mathrm{p}$.

Verneaux (J.). 1973. $\rightarrow$ Cours d'eau de France Comté (Massif du Jura). Recherches écologiques sur le réseau hydrographique du Doubs - Essai de biotypologie. Annls Scient. Univ. Besançon, Zool. Physiol. Biol. anim., 3 (9) : 260 p.

Wachs (B.). 1967. - Die Oligochaeten-Fauna der Fliessgewässer unter besonderer Berücksichtigung der Beziehungen zwischen der Tubificiden-Besiedlung und dem Substrat. Arch. Hydrobiol, 63 (3) : 310-386.

Young (J.O.). 1974. - Life-cycles of some Invertebrate taxa in a small pond with changes in their numbers over a period of three years. Hydrobiologia, 45 (I) : 63-90. 